\title{
Kôm Ombo (2020)
}

\section{Françoise Labrique et Ali Abdelhalim Ali}

\section{OpenEdition \\ Journals}

Édition électronique

URL : https://journals.openedition.org/baefe/3093

DOI : $10.4000 /$ baefe.3093

ISSN : 2732-687X

Éditeur

ResEFE

\section{Référence électronique}

Françoise Labrique, Ali Abdelhalim Ali, « Kôm Ombo (2020) » [notice archéologique], Bulletin

archéologique des Écoles françaises à l'étranger [En ligne], Égypte, mis en ligne le 30 mai 2021, consulté le 27 juillet 2021. URL : http://journals.openedition.org/baefe/3093 ; DOI : https://doi.org/10.4000/

baefe.3093

Ce document a été généré automatiquement le 27 juillet 2021.

\section{cc) (†) $\odot$}

Le Bulletin archéologique des Écoles françaises à l'étranger est mise à disposition selon les termes de la Licence Creative Commons Attribution - Pas d'Utilisation Commerciale - Pas de Modification 4.0 International. 


\title{
Kôm Ombo (2020)
}

\author{
Françoise Labrique et Ali Abdelhalim Ali
}

\section{NOTE DE L'AUTEUR}

Année de la campagne : 2020 ( 7 octobre - 5 novembre)

Autorité nationale présente : Le ministère du Tourisme et des Antiquités (MoTA) était représenté par Mustafa Muhammed (inspecteur, inspectorat de Kôm Ombo) et restaurateur Emad Abdelaziz (restaurateur, inspectorat de Kôm Ombo)

Numéro et intitulé de l'opération de terrain : 17151 - Kôm Ombo. Le temple de Haroëris et Sobek à Kôm Ombo : relevés photographiques, mission épigraphique, préservation du site

Composition de l'équipe de terrain : L'équipe de terrain était composée de Françoise Labrique (Universität zu Köln), Shafia Bedier (égyptologue, université Ayn Shams), Ali Abdelhalim Ali (égyptologue, université Ayn Shams), Islam Alwakeel (égyptologue, université Ayn Shams), Gaël Pollin (photographe, Ifao), Mohammed Abdu (intendant de chantier, Ifao), Muhammed Husein (ouvrier, Gourna), Muhammed Qaed (ouvrier, Gourna), Mustafa Qaed (ouvrier, Gourna), Waled Shafie (ouvrier, Beni Souef), Karam Ahmed (ouvrier, Kôm Ombo) et Saleh Mahmoud (ouvrier, Kôm Ombo).

Partenariats institutionnels : La mission bénéficie du soutien de l'Universität zu Köln et du l'université Ayn Chams.

Organismes financeurs : Deutsche Forschungsgemeinschaft

1 Le programme prévu pour la mission d'octobre 2020 a été réadapté aux circonstances affectées par la pandémie.

2 1. Sous la direction d'Ali Abdelhalim Ali, l'équipe, encadrée par Mohamed Abdou, a poursuivi la construction des mastabas dans le secteur nord du complexe du temple de Haroëris et Sobek, entamée en 2019, en ajoutant quatre socles nouveaux. La section lapidaire comprend à présent quatre mastabas longs de $400 \times 100 \times 40 \mathrm{~cm}$ et quatre petits socles de $100 \times 100 \times 40 \mathrm{~cm}$. 
2. Plusieurs pierres, la plupart inscrites, ont été disposées sur les socles, parmi elles, un bloc, partie d'une statue colossale de Ramsès II (fig. 1), a été exposé sur la construction centrale. La répartition est temporaire, notre équipe prévoyant lors d'une saison prochaine de classer et étiqueter les objets exposés (fig. 2).

Fig. 1. Halage de la statue de Ramsès II (A. Abdelhalim Ali, I. Alwakeel).

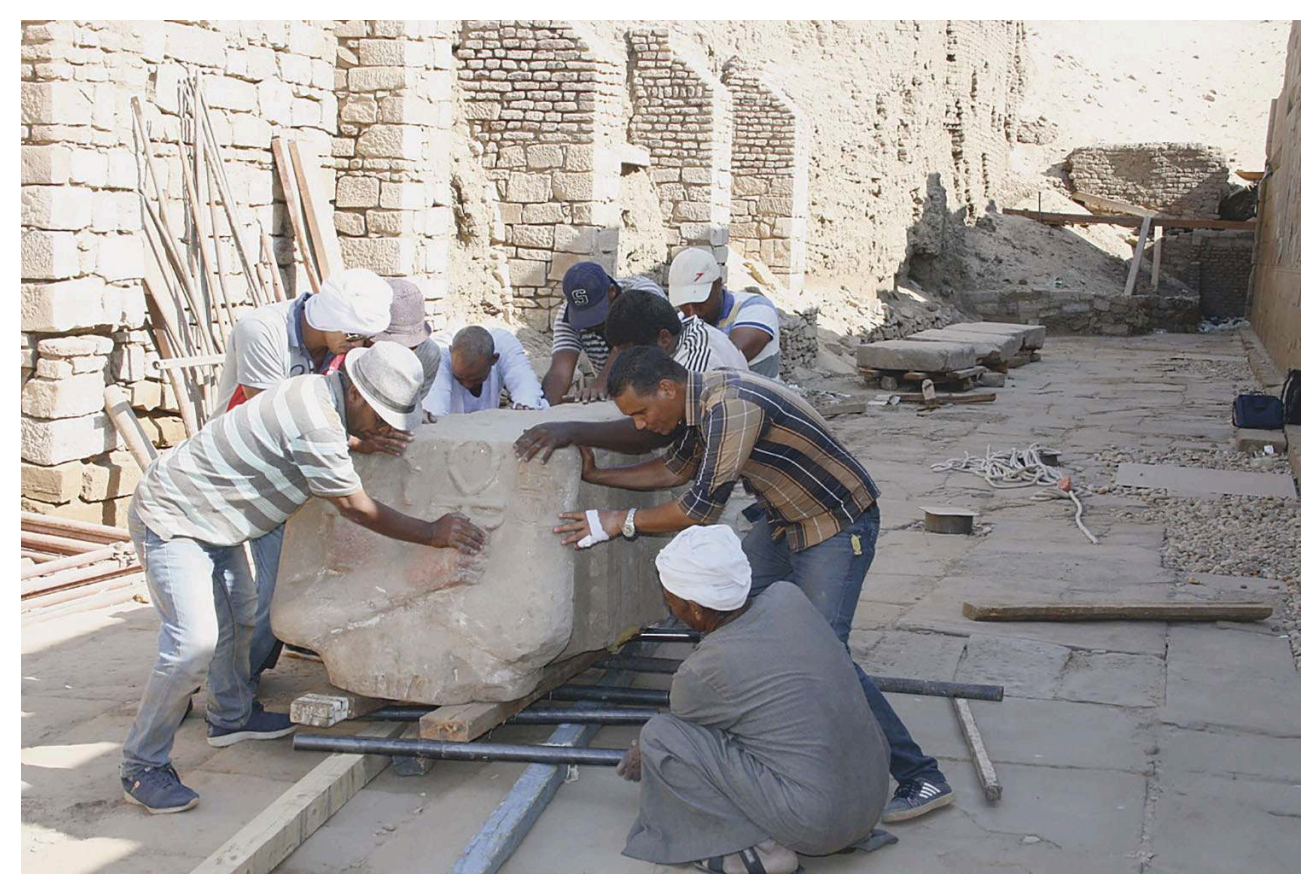

(c) Ifao. 17151_2020_NDMPF_005.

Fig. 2. Exposition provisoire de pierres éparses(A. Abdelhalim Ali, I. Alwakeel).

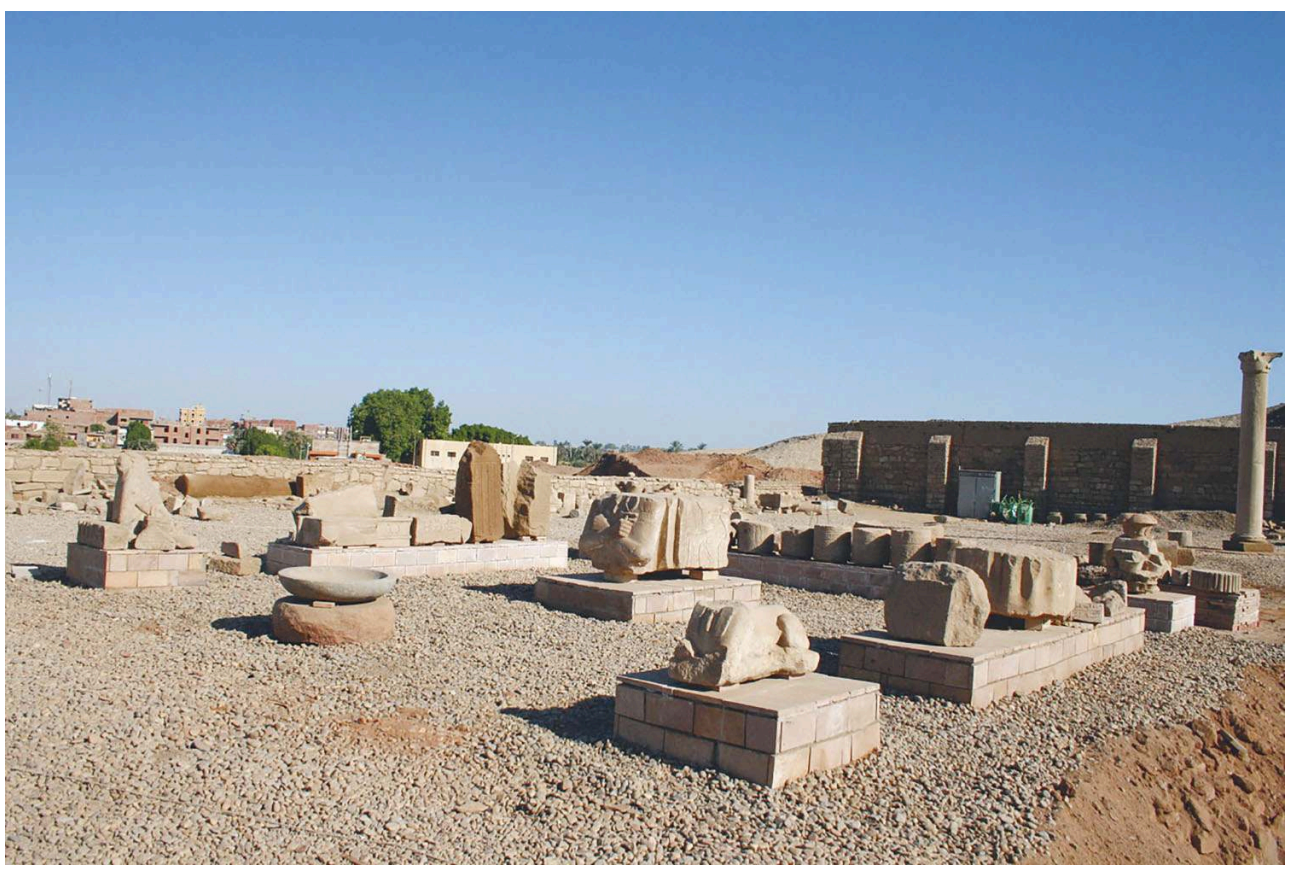

(C) Ifao. 17151_2020_NDMPF_007. 
4 3. Parmi les blocs entassés sur le sol dans le secteur situé au nord du temple majeur (fig. 3-4), Ali Abdelhalim Ali a repéré un siège de prêtre, qui gisait retourné et a été disposé sur un des nouveaux mastabas (fig. 5). Ce siège s'apparente à trois autres encore in situ, alignés à droite de l'entrée de la chapelle de Sobek, dédiée par Caracalla et Géta et découverte par Barsanti en 1914. Ces trois objets ont été comparés par Laurent Coulon en 2006 à un groupe de sièges de prêtres de Karnak ${ }^{1}$.

Fig. 3. Secteur au nord du temple majeur, partie ouest (A. Abdelhalim Ali).

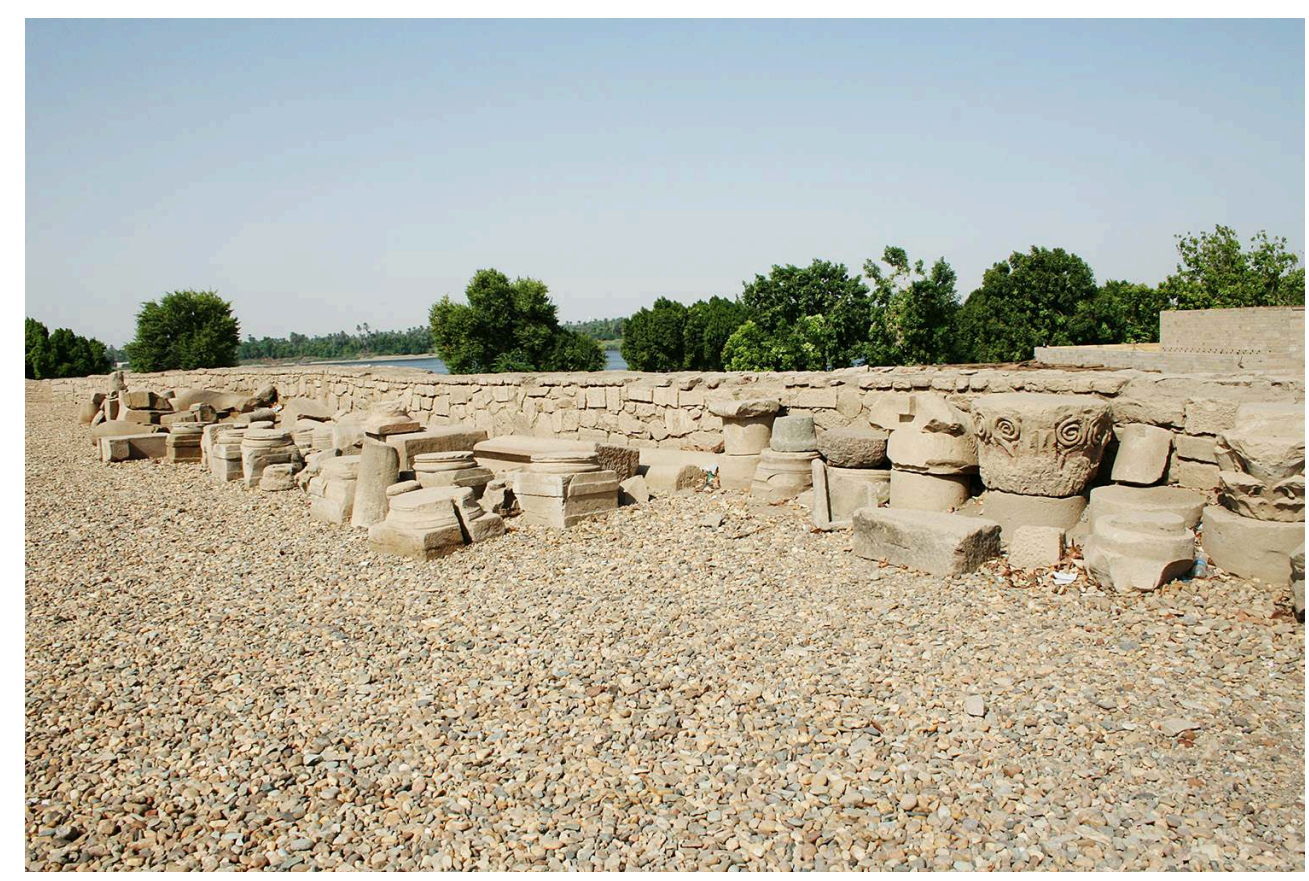

(C) Ifao. 17151_2020_NDMPF_009 
Fig. 4. Secteur au nord du temple majeur, partie est (A. Abdelhalim Ali).

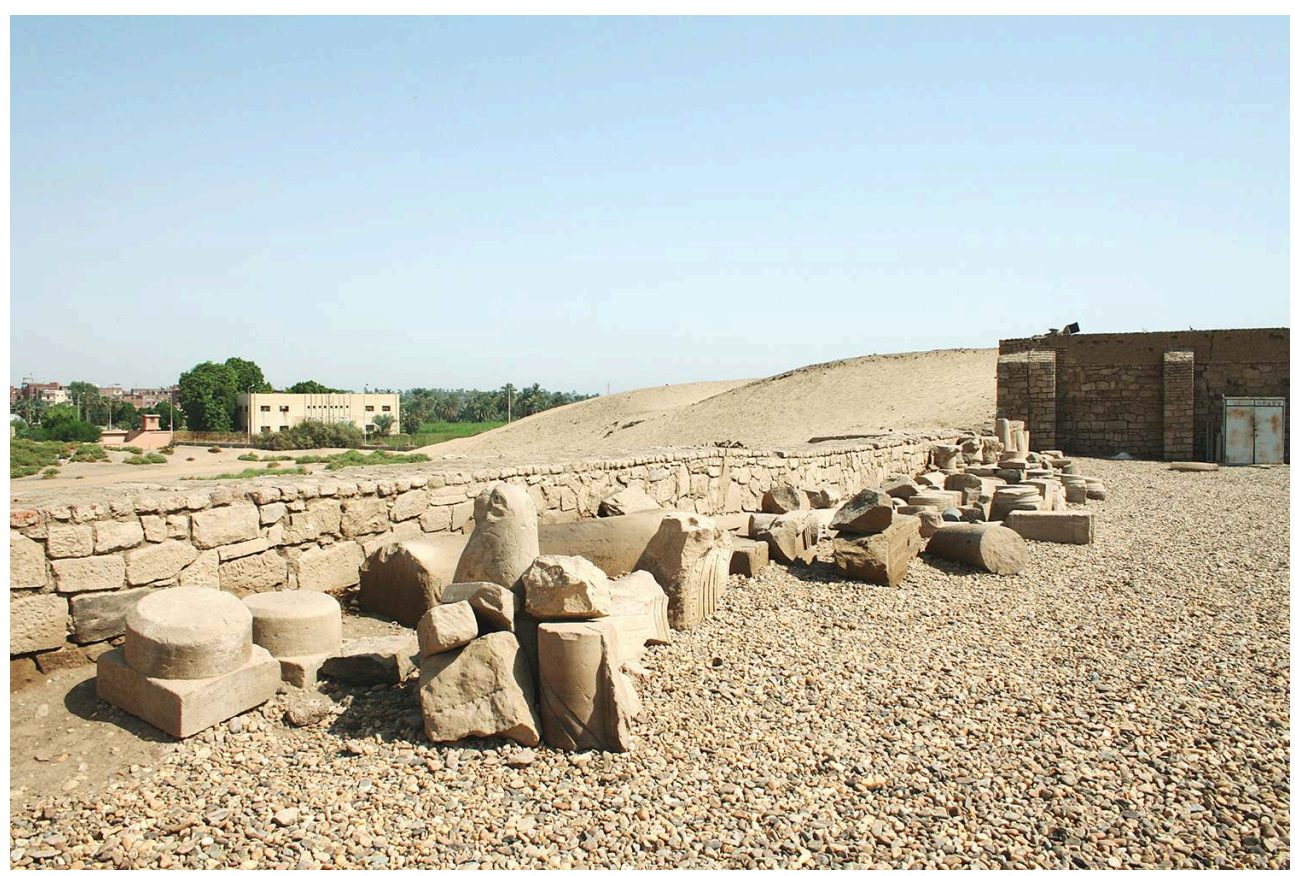

(c) Ifao. 17151_2020_NDMPF_010

Fig. 5. Un siège de prêtre (A. Abdelhalim Ali, I. Alwakeel).

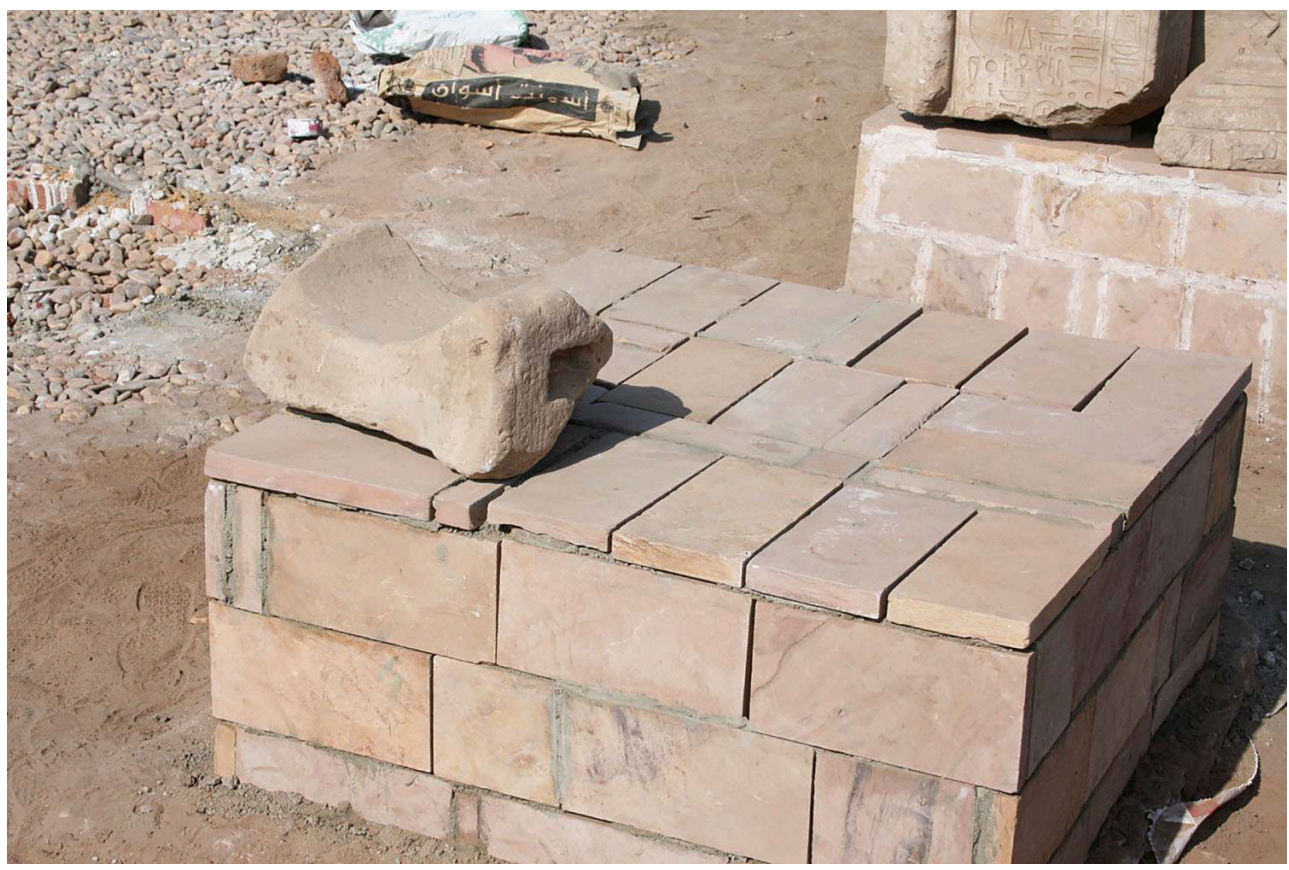

(c) Ifao. 17151_2020_NDMPF_004

5 4. Privé cette année des grands échafaudages de la maison américaine d'Abydos, Gaël Pollin a travaillé sur des surfaces plus aisément accessibles que les hauteurs du pronaos (dont la documentation est progressivement constituée pour préparer la publication de Kôm Ombo IV). Il a photographié intégralement deux monuments : 
4.1. Le mammisi : les clichés nu_2020_04536-04573 sont l'objet d'une étude par Ali Abdelhalim Ali, qui prépare la réédition de ce monument et a procédé à l'examen épigraphique des textes en les transcrivant à l'aide du programme JSesh, dans le cadre d'une recherche menée sous l'égide de l'Ifao.

4.2. La chapelle de Sobek, datée de Caracalla et de Géta (nu_2020_04578 et nu_2020_04587) : Ali Abdel Halim Ali en prépare l'édition dans le cadre d'une recherche menée sous l'égide de l'ARCE.

4.3. Gaël Pollin a également commencé le relevé photographique du pronaos en couvrant les deux portes latérales de la façade (nu_2020_04574 et nu_2020_04577) :

- La petite porte sud, extérieur, correspondant au n 43 du PM VI, 183 et aux tableaux 193-194 de l'édition de J. de Morgan en 1895 (disposition des tableaux p. 136). Le linteau (tableau 194) contient une monographie étudiée par Adolpe Gutbub (1973, p. 66-85).

- La petite porte nord, extérieur, correspondant au $n^{\circ} 41$ du PM VI, 183 et aux tableaux 181-182 de l'édition de J. de Morgan, Kom Ombos II, $1^{\text {re }}$ partie, (disposition des tableaux p.136). Le linteau (tableau 182) contient une monographie étudiée par A. Gutbub (1973, p. 57-66).

- La petite porte sud, intérieur, correspondant au $\mathrm{n}^{\circ} 44 \mathrm{du}$ PM VI, 183 et aux tableaux 216-217 de l'édition de J. de Morgan, Kom Ombos II, $1^{\text {re }}$ partie, (disposition des tableaux p.158). Le linteau (tableau 217) représente Ptolémée XII offrant Heh aux quatre vents.

- La petite porte nord, intérieur, correspondant au $n^{\circ} 44$ du PM VI, 183 et aux tableaux 210-211 de l'édition de J. de Morgan, Kom Ombos II, $1^{\text {re }}$ partie, (disposition des tableaux p.158). Le linteau (tableau 211) représente Ptolémée XII et Cléopâtre VI offrant un plateau d'aliments et deux gerbes florales à une triade réunissant Haroéris seigneur d'Ombos, Sobek-Rê seigneur de Ta-Sety et Tasenteneferet maîtresse d'Ombos.

Fig. 6. Le mammisi de Kôm Ombo, vu de l'est (A. Abdelhalim Ali, I. Alwakeel).

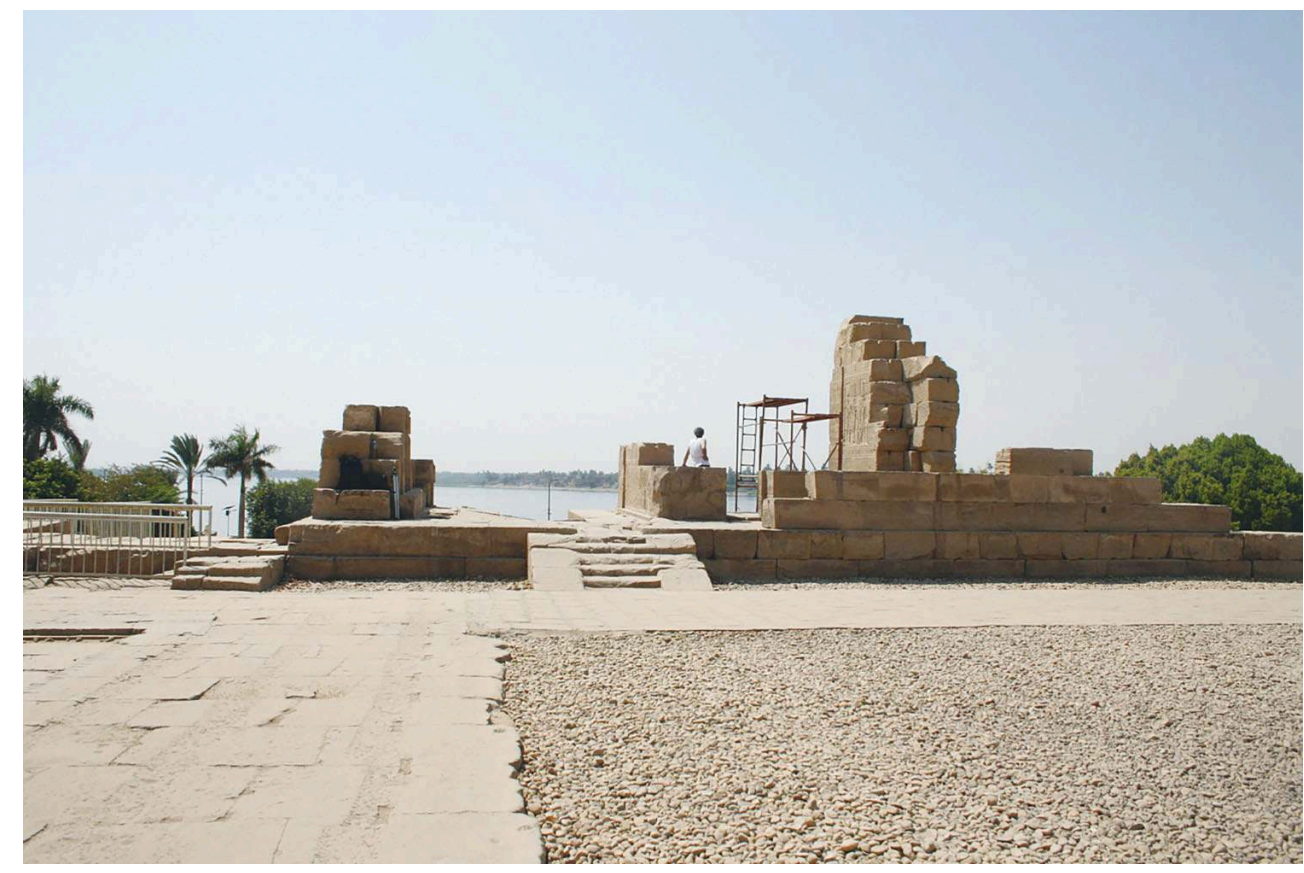

(c) Ifao. 17151_2020_NDMPF_003 
6 5. Les inscriptions du mammisi (fig. 6), dont la publication est préparée par Ali Abdelhalim Ali, et des colonnes de la petite salle hypostyle, objet du futur Kôm Ombo III, ont été collationnées.

7 6. Le sol à l'arrière du mammisi a été consolidé, pour y empêcher l'écroulement des dalles branlantes (fig. 7).

Fig. 7. Parties du mammisi à étayer (A. Abdelhalim Ali, I. Alwakeel).

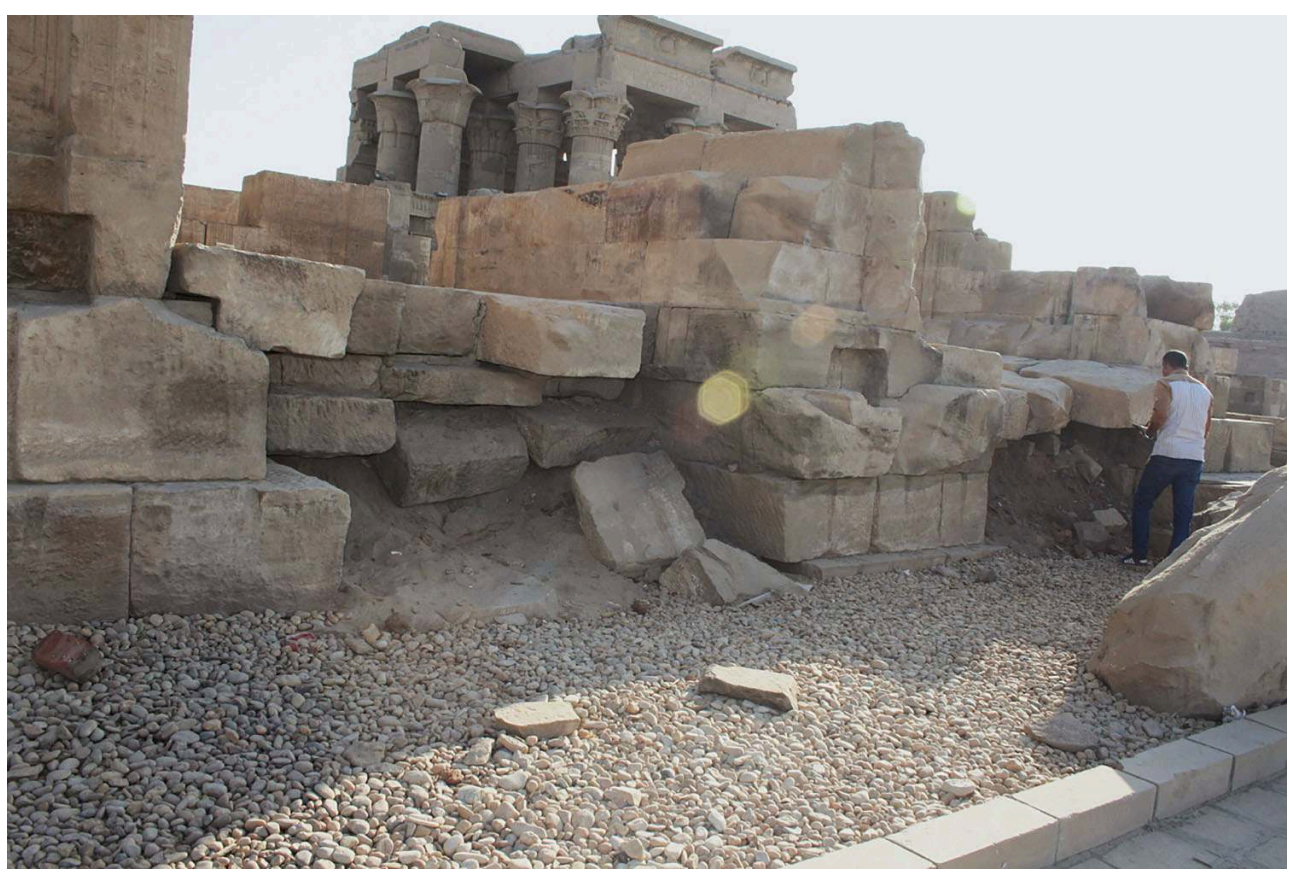

(c) Ifao. 17151_2020_NDMPF_006

8 7. À la demande de l'inspectorat de Kôm Ombo, un panneau a été dressé dans le secteur nord, expliquant en arabe et en anglais la fameuse scène aux instruments chirurgicaux (fig. 8). Il contient une photographie réalisée par Gaël Pollin et un commentaire rédigé par Ali Abdelhalim Ali. 
Fig. 8. Panneau relatif aux instruments chirurgicaux (A. Abdelhalim Ali, I. Alwakeel).

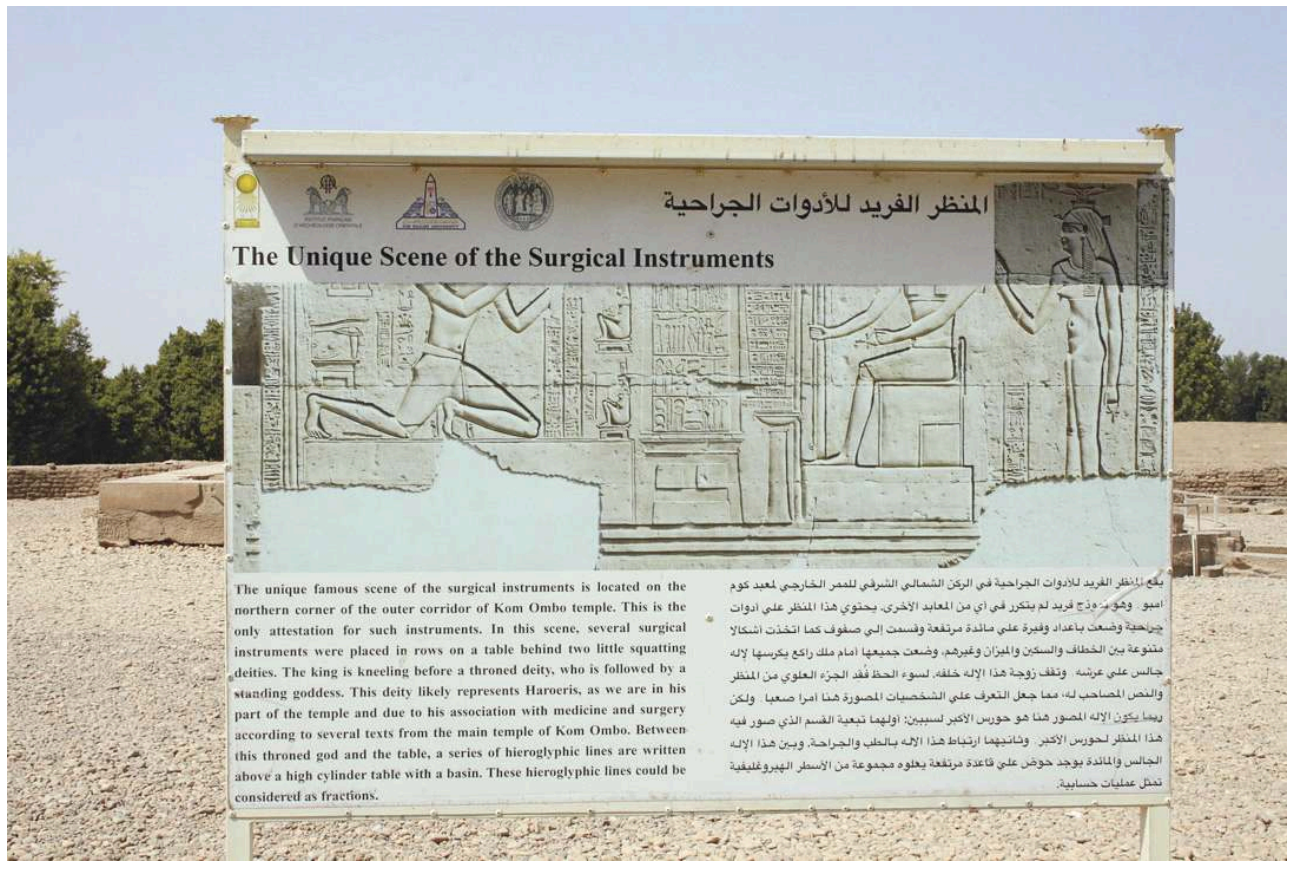

(C) Ifao. 17151_2020_NDMPF_008

\section{BIBLIOGRAPHIE}

COULON 2006

Laurent Coulon, «Les sièges de prêtre d'époque tardive. À propos de trois documents thébains ", $R d E$ 57, 2006, p. 1-31

DE MORGAN 1895

Jacques De Morgan, Catalogue des Monuments et inscriptions de l'Égypte antique, vol. 2 : Kom Ombos, première partie, Vienne, Holzhausen, 1895.

GutBuB 1973

Adolphe Gutbub, Texte Fondamentaux de la théologie de Kom Ombo, BdE 47/1, Le Caire, Ifao 1973.

\section{NOTES}

1. COULON 2006. 
INDEX

Thèmes : IFAO

chronologie https://ark.frantiq.fr/ark:/26678/pcrtM6FrOydySh

nature https://ark.frantiq.fr/ark:/26678/pcrtPjg2s77qPX

Année de l'opération : 2020

sujets https://ark.frantiq.fr/ark:/26678/pcrtYhYMiLwDUr, https://ark.frantiq.fr/ark:/26678/

pcrtPjg2s77qPX, https://ark.frantiq.fr/ark:/26678/pcrtRXhdi405ST

\section{AUTEURS}

\section{FRANÇOISE LABRIQUE}

Universität zu Köln

ALI ABDELHALIM ALI

Égyptologue, université Ayn Shams 